\title{
OPTIMUM LAG AND SUBSET SELECTION FOR A RADIAL BASIS FUNCTION EQUALISER
}

\author{
E.S.CHNG ${ }^{\dagger}$, B.MULGREW $^{\dagger}$, S.CHEN ${ }^{\ddagger}$ and G.GIBSON ${ }^{+}$ \\ $\dagger$ Dept. of Electrical Eng., The University of Edinburgh, U.K. \\ \$ Dept. of Electrical and Electronic Eng., The University of Portsmouth, U.K \\ + Scottish Agricultural Statistics Service, The University of Edinburgh, U.K
}

\begin{abstract}
This paper examines the application of the radial basis function (RBF) network to the modelling of the Bayesian equaliser. In particular, we study the effects of delay order $d$ on decision boundary and attainable bit error rate (BER) performance. To determine the optimum delay parameter for minimum BER performance, a simple BER estimator is proposed.

The implementation complexity of the RBF network grows exponentially with respect to the number of input nodes. As such, the full implementation of the RBF network to realise the Bayesian solution may not be feasible. To reduce some of the implementation complexity, we propose an algorithm to perform subset model selection. Our results indicate that it is possible to reduce model size without significant degradation in BER performance.

Indexing Term: Bayesian equaliser, neural networks, RBF network, BER.
\end{abstract}

\section{Introduction}

It is well-known that the performance of neural network (NN) equaliser is superior to the conventional linear equaliser for the digital communication symbol-by-symbol equalisation problem [1-3]. The superiority of the NN structure is due to its ability to model the optimum Bayesian decision boundary better than the conventional linear systems. In many practical equalisation problems, the Bayesian decision boundary is often highly nonlinear, and in some cases, not linearly separable. It is thus not surprising that NN techniques, which are capable of modelling any nonlinear decision boundaries, have become very popular in equalisation problems. This paper continues this theme and investigates the application of the radial basis function (RBF) network to realise the Bayesian equaliser.

The paper is organised as follows: In Sec. 2.1, we extend the work reported in $[1,2]$ to show the effects of delay order on the Bayesian equaliser's decision boundary and BER performance. Our analysis show that the equaliser achieves different attainable BER performance when different delay order

0-7803-2739-X/95 \$4.00 ㄷ 1995 IEEE 
is applied under the same signal to noise (SNR) operating condition. To determine the optimum delay order, a simple BER estimate for the equaliser is proposed in Sec. 3. The implementation complexity of the RBF equaliser is also discussed, and an algorithm to select small-sized RBF models which approximate the Bayesian solution is presented in Sec.4

\section{Implementing the Bayesian equaliser}

An established model of a digital communication channel subjected to intersymbol interference (ISI) for a multi-level pulse amplitude modulation (2-ary PAM) scheme is described by the following equation $[2,4]$ :

$$
r(k)=\sum_{i=0}^{n_{a}-1} s(k-i) a(i)+e(k)
$$

where $r(k)$ is the received signal at time $k, s(k)$ is an independently identically distributed (i.i.d) transmitted symbol with symbol constellation defined by the set $\{ \pm 1\}, a(i)$ are the channel impulse response coefficients with the length of the impulse response $n_{a}$, and $e(k)$ is the additive white Gaussian noise $e(k)$ of zero mean and variance $\sigma_{e}^{2}[2,4]$. The equaliser uses an array of received signal

$$
\mathbf{r}(k)=[r(k), \cdots, r(k-m+1)]^{T}
$$

to estimate the transmitted symbol $s(k-d)$, i.e. $\hat{s}(k-d)$. The integers $m$ and $d$ are known as the feedforward order and delay order respectively.

The transmitted symbols that affect the input vector $\mathbf{r}(k)$ is the transmit sequence $\mathbf{s}(k)=\left[s(k), \cdots, s\left(k-m-n_{a}+2\right]^{T}\right.$. There are $N_{s}=2^{m+n_{a}-1}$ possible combinations of these input sequences, i.e. $\left\{\mathbf{s}_{j}\right\}, 1 \leq j \leq N_{s}$ [2]. In the absence of noise, there are $N_{s}$ corresponding received sequences $C_{d}=$ $\left\{\mathrm{c}_{j}\right\}, 1 \leq j \leq N_{s}$, which are also referred to as channel states. The subscript $d$ in $C_{d}$ denotes the delay order used. The values of the channel states are defined by the following equation,

$$
\mathrm{c}_{j}=F\left[\mathbf{s}_{j}\right] \quad 1 \leq j \leq N_{s}
$$

where the matrix $F \in R^{m \times\left(m+n_{a}-1\right)}$ is

$$
F=\left[\begin{array}{cccccccc}
a(0) & a(1) & \ldots & a\left(n_{a}-1\right) & 0 & \ldots & \ldots & 0 \\
0 & a(0) & a(1) & \ldots & a\left(n_{a}-1\right) & 0 & \ldots & 0 \\
\vdots & \vdots & \vdots & \vdots & \vdots & \vdots & \vdots & \vdots \\
0 & \ldots & \ldots & \ldots & a(0) & a(1) & \ldots & a\left(n_{a}-1\right)
\end{array}\right]
$$

When noise is present, the received vector $\mathbf{r}(k)$ has a Gaussian distribution with expected values corresponding to the respective $\mathbf{c}_{j}$.

The set of channel states $\left\{\mathbf{c}_{j}\right\}, 1 \leq j \leq N_{s}$ can be partitioned according to the value of $s(k-d)$, i.e., channel states associated with $s(k-d)=+1$ 
belong to the class $C_{d}^{(+)}$, and channel states associated with $s(k-d)=-1$ belong to the class $C_{d}^{(-)}$. The response of the Bayesian equaliser prior to the slicer is [2],

$$
\begin{aligned}
f(\mathbf{r}(k))= & \sum_{\mathbf{c}_{i} \in C^{(+)}} p_{i}\left(2 \pi \sigma_{e}^{2}\right)^{-m / 2} \exp \left(-\left\|\mathbf{r}(k)-\mathbf{c}_{i}\right\|^{2} / 2 \sigma_{e}^{2}\right) \\
& -\sum_{\mathbf{c}_{j} \in C^{(-)}} p_{j}\left(2 \pi \sigma_{e}^{2}\right)^{-m / 2} \exp \left(-\left\|\mathbf{r}(k)-\mathbf{c}_{j}\right\|^{2} / 2 \sigma_{e}^{2}\right)
\end{aligned}
$$

where $p_{i}$ and $p_{j}$ are the a priori probabilities of occurrence for the respective channel states. In the case of i.i.d transmitted symbols, $p_{i}=p_{j}=1 / N_{s}$. The output of the Bayesian equaliser $\hat{s}(k-d)$ is $\operatorname{sgn}(f(\mathbf{r}(k))$, where $\operatorname{sgn}($.$) is the$ signum function.

From Eq. 5, it is obvious that the structure of the RBF network is identical to the Bayesian equaliser [2], and that the RBF network realises precisely the Bayesian solution when the weights, centres and the nonlinearity of hidden units are set accordingly.

\subsection{Effects of delay order on decision boundaries}

The set $\{\mathbf{r}(k) \mid f(\mathbf{r}(k))=0\}$ defines the Bayesian decision boundary and is dependent on the channel state values and the delay order parameter $[1,2]$. The channel states are determined by the channel impulse response and the equaliser feedforward order. The channel states however do not uniquely define the decision boundary. Given a set of channel states, the decision boundary can be changed by using different delay orders.

As an example, the Bayesian decision boundaries realised by a RBF equaliser with feedforward order $m=2$ for channel $H(z)=0.5+1.0 z^{-1}$ is examined. Fig 1a lists all the 8 possible combinations of the transmitted signal sequence $\mathbf{s}(k)$ and the corresponding channel states $\mathbf{c}_{i}$. Fig. $1 \mathrm{~b}$ depicts the corresponding decision boundaries for the different delay orders. Note the dramatic change in the shape of the decision boundaries for different delay orders.

The use of different delay orders also results in different limits of BER performance. To determine the optimum delay order, a computationally simple method to estimate the BER of the Bayesian equaliser is presented in Sec. 3.2.

\section{Probability of mis-classification}

This section presents the analysis of probability of mis-classification of the Bayesian equaliser. 


\begin{tabular}{|c|c|c|c|c|c|}
\hline \multirow[t]{2}{*}{$\begin{array}{c}\mathrm{S} / \mathrm{No} \\
i\end{array}$} & \multicolumn{3}{|c|}{$\begin{array}{c}\text { Transmitted symbols } \\
\mathbf{s}(k)\end{array}$} & \multicolumn{2}{|c|}{$\begin{array}{c}\text { Channel State } \\
\mathbf{c}_{i}\end{array}$} \\
\hline & {$[s(k)$} & $s(k-1)$ & $s(k-2)]$ & {$[r(k)$} & $r(k-1)]$ \\
\hline 1 & 1 & 1 & 1 & 1.5 & 1.5 \\
\hline 2 & 1 & 1 & -1 & 1.5 & -0.5 \\
\hline 3 & 1 & -1 & 1 & -0.5 & 0.5 \\
\hline 4 & 1 & -1 & -1 & -0.5 & -1.5 \\
\hline 5 & -1 & 1 & 1 & 0.5 & 1.5 \\
\hline 6 & -1 & 1 & -1 & 0.5 & -0.5 \\
\hline 7 & -1 & -1 & 1 & -1.5 & 0.5 \\
\hline 8 & -1 & -1 & -1 & -1.5 & -1.5 \\
\hline
\end{tabular}

Fig (a) : State Table

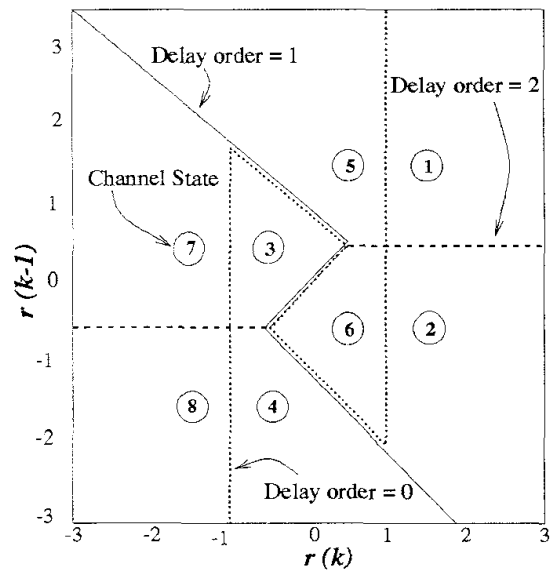

Fig (b) : Decision boundaries

Figure 1: (a) Input and desired channel states for channel $H(z)$, (b) Bayesian decision boundaries for channel $H(z)$.

\subsection{Evaluating the probability of error}

We define $Z^{+} \subset R^{m}$ to be the region of $\mathbf{r}(k)$ classified as +1 and $Z^{-} \subset R^{m}$ to be the region classified as -1 . The probability of making a wrong decision $P_{e}$ is

$$
P_{e}=\sum_{c_{i} \in \mathbf{C}^{+}} p_{i} \int_{\mathbf{r} \in Z^{-}} f_{\mathbf{r} \mid \mathbf{c}_{i}}(\mathbf{r}) d \mathbf{r}+\sum_{\mathbf{c}_{j} \in \mathbf{c}^{-}} p_{j} \int_{\mathbf{r} \in Z^{+}} f_{\mathbf{r} \mid \mathbf{c}_{j}}(\mathbf{r}) d \mathbf{r}
$$

where $f_{\mathbf{r} \mid \mathbf{c}_{l}}(\mathbf{r})$ is the probability density function (pdf) of the noisy received vector $\mathbf{r}$ conditioned on the received channel state being $c_{l}$,

$$
f_{\mathbf{r} \mid \mathbf{c}_{l}}(\mathbf{r})=\left(2 \pi \sigma_{e}^{2}\right)^{-m / 2} \exp \left(-\left\|\mathbf{r}-\mathbf{c}_{l}\right\|^{2} /\left(2 \sigma_{e}^{2}\right)\right)
$$

Because the symbol constellation is symmetric, equation (6) can be reduced to

$$
P_{e}=2 \sum_{\mathbf{c}_{i} \in \mathbf{c}^{+}} p_{i} \int_{\mathbf{r} \in Z^{-}} f_{\mathbf{r} \mid \mathbf{c}_{i}}(\mathbf{r}) d \mathbf{r}
$$

\subsection{Estimating the probability of error}

The evaluation of BER using Eq. 8 involves evaluating $m$-dimensional integrals over the error region $Z^{-}$. As a closed-form solution for the expression does not exist, one must resort to numerical methods. This option however 
is un-attractive for large $m$. As our requirement to find BER performance is only one of comparing relative performance for equalisers using different delay orders, a simple approximation may be used to estimate the BER. The probability of mis-classifications, $P_{e}$, can be expressed as

$$
P_{e}=\frac{1}{N_{s}} \sum_{i=1}^{N_{s}} P_{e}\left(\mathbf{c}_{i}\right)
$$

where $P_{e}\left(\mathbf{c}_{i}\right)$ is the probability of mis-classification conditioned on the noisefree channel state being $\mathbf{c}_{i}$. It can be shown that in the case when SNR $\rightarrow \infty$, $P_{e}\left(\mathbf{c}_{i}\right)$ can be reduced to the minimum distance bound [5], i.e.,

$$
P_{e}\left(\mathbf{c}_{i}\right)=Q\left(\left|\zeta_{i}\right| / \sigma_{e}\right)=\int_{\left|\zeta_{i}\right|}^{\infty}\left(2 \pi \sigma_{e}^{2}\right)^{-1 / 2} \exp \left(-x^{2} /\left(2 \sigma_{e}^{2}\right)\right) d x
$$

where $\left|\zeta_{i}\right|$ is the absolute minimum Euclidean distance of $\mathbf{c}_{b}$ to the decision boundary.

Although Eq. 10 is only valid for very high SNR, it can be applied with Eq. 9 to evaluate a rough estimate of the BER performance. Our simulation results however indicate that the proposed estimator gives good BER estimates even for low SNRs.

\subsection{BER estimate : Some simulation results}

Simulations were conducted to compare the BER results obtained using Eqs. 9 and 10 with those obtained by the Monte Carlo (MC) simulations. The following channels which have the same magnitude but different phase response were used,

$$
\begin{aligned}
& H 1(z)=0.8745+0.4372 z^{-1}-0.2098 z^{-2} \\
& H 2(z)=0.2620-0.6647 z^{-1}-0.2623 z^{-2}
\end{aligned}
$$

For the experiment, the equaliser's feedforward order was chosen to be 4 with the transmit symbol alphabet $\{ \pm 1\}$. Fig 2 compares the BER estimates of Eqs. 9 and 10 with those of MC simulations for the two channels using different delay orders. The results show that the proposed BER estimate is very accurate. 'To illustrate the strong dependence of the equaliser's performance with respect to the delay order, we plot the performance of the equaliser using the delay parameter as the horizontal axis in $\mathrm{Fig} 3$.

\section{Selecting subset RBF model}

The implementation of the full RBF solution requires the use of all $N_{s}$ channel states. In some cases, equivalent Bayesian solution may be realised by using a subset of the full model. For example, the decision boundaries of delay 

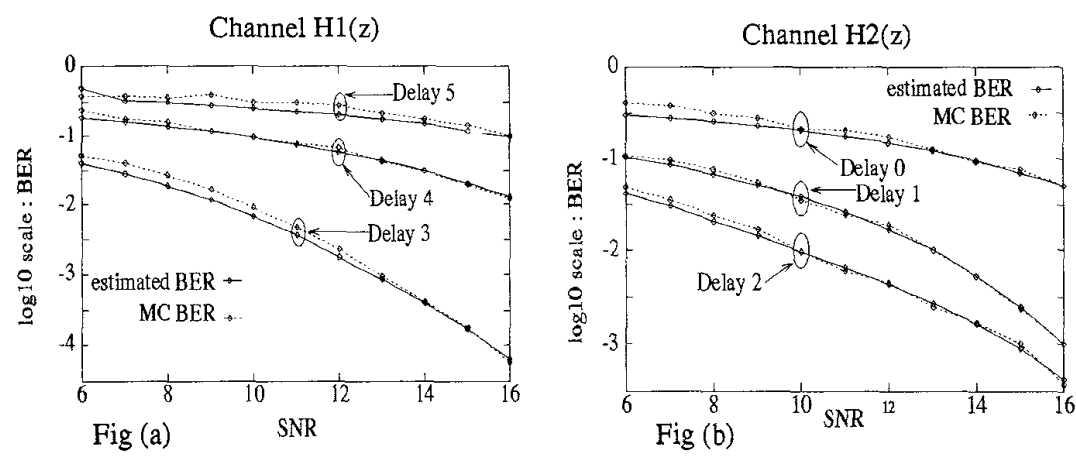

Figure 2: Estimated and $\mathrm{MC}$ simulations of BER vs SNR for $H 1(z)$ (Fig a) and $H 2(z)$ (Fig b).

order 1 and 2 (Fig $1 \mathrm{~b}$ ) can be realised by a RBF model with centres $c_{i}$ from $\left\{\mathbf{c}_{3}, \mathbf{c}_{4}, \mathbf{c}_{5}, \mathbf{c}_{6}\right\}$ (Fig. 4a,b).

In many cases, we have observed that it is possible to find small subset RBF model to approximate the full model's solution when the decision boundary is linearly separable. The task is however much more difficult when the decision boundary is nonlinearly separable.

This section examines subset model selection algorithms to reduce implementation complexity of the RBF equaliser. The objective is to find a smaller-sized, in terms of number of centres, RBF model to realise or to approximate the same Bayesian solution as the full model. To understand how centres affect boundary, we analyse the effects of centre positions on decision boundary when $\sigma_{e} \rightarrow 0$. Defining the points on the boundary as $\mathbf{r}_{0}$, i.e. $\left\{\mathbf{r}_{0} \mid f\left(\mathbf{r}_{0}\right)=0\right\}$, Eq. 5 becomes

$$
\begin{gathered}
\sum_{\mathbf{c}_{i} \in C_{d}^{(+)}} p_{i}\left(2 \pi \sigma_{e}^{2}\right)^{-m / 2} \exp \left(-\left\|\mathbf{r}_{0}-\mathbf{c}_{i}^{+}\right\|^{2} / 2 \sigma_{e}^{2}\right)= \\
\sum_{\mathbf{c}_{j} \in C_{d}^{(-)}} p_{j}\left(2 \pi \sigma_{e}^{2}\right)^{-m / 2} \exp \left(-\left\|\mathbf{r}_{0}-\mathbf{c}_{j}^{-}\right\|^{2} / 2 \sigma_{e}^{2}\right)
\end{gathered}
$$

When $\sigma_{e} \rightarrow 0$, the sum on the 1.h.s. of Eq. 13 becomes dominated by the closest centres to $\mathbf{r}_{0}$, i.e.

$$
U^{+}=\min _{\mathbf{c}_{k} \in C_{d}^{(+)}}\left\{\left\|\mathbf{r}_{0}-\mathbf{c}_{k}\right\|\right\}
$$

This is because the contribution of centres $\mathbf{c}_{k} \notin U^{+}$converges much more quickly to zero when $\sigma_{e} \rightarrow 0$ than centres belonging to $U^{+}$. Similarly, the sum on the r.h.s of Eq. 13 becomes dominated by the closest centres, $U^{-}$. This 

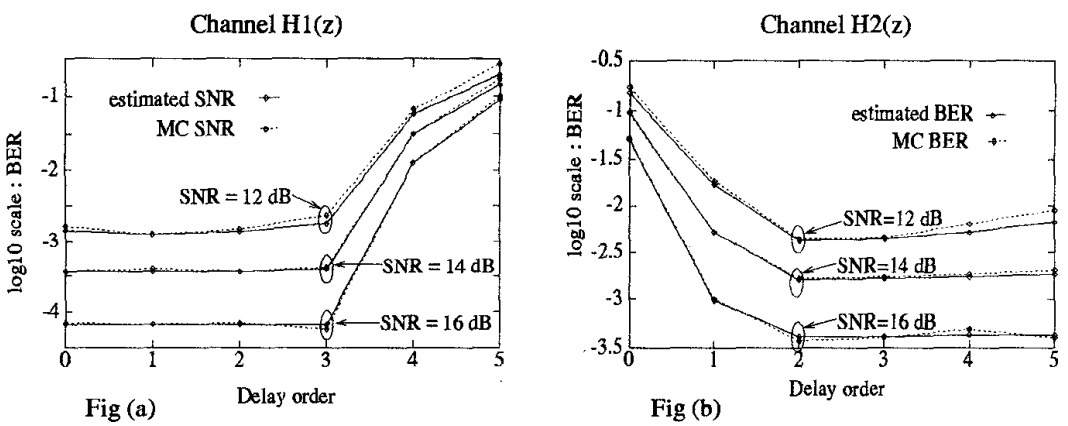

Figure 3: Estimated and MC simulations BER vs delay order for SNR 12dB, $14 \mathrm{~dB}$ and $16 \mathrm{~dB}$ for $H 1(z)$ (Fig a) and $H 2(z)$ (Fig b).

implies that the asymptotic decision boundaries are hyper-planes between pairs of $U^{+}, U^{-}$and that the set of all $U^{+}, U^{-}$defines the asymptotic decision boundaries. The following algorithm may be employed to find the set of all $U^{+}, U^{-}$.

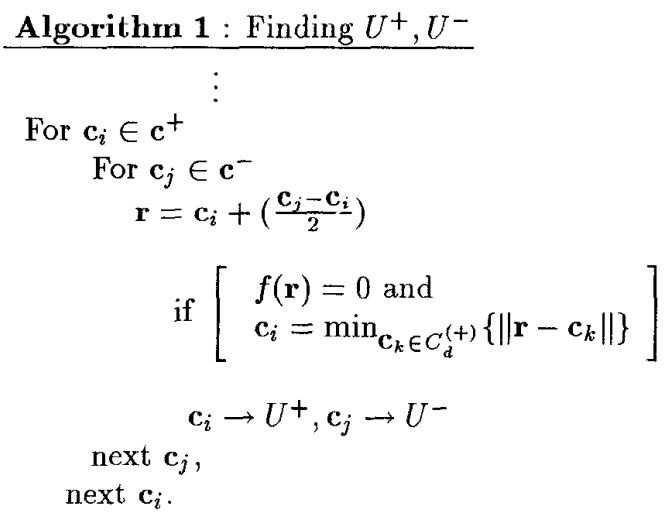

Algorithm 1 was tested to find subset models from the full RBF model (Sec. 2.1) used on channel $H(z)=0.5+1.0 z^{-1}$. As expected, when delay order 0 was used, all the centres, $\left\{\mathbf{c}_{1}, \ldots, \mathbf{c}_{8}\right\}$ were picked to form the subset model (Fig. 1b). For the case of using delay order 1, the selected subset model consisted of centres $\left\{\mathbf{c}_{3}, \mathbf{c}_{4}, \boldsymbol{c}_{5}, \mathbf{c}_{6}\right\}$ These results can be easily verified by visual inspection of the boundary formation as illustrated in Figs. 4a and $1 \mathrm{~b}$.

Although algorithm 1 works, the selection process is not optimum in the sense that redundant centres may be included to form the subset model. To illustrate, consider the selected subset model when delay order 2 was used. 

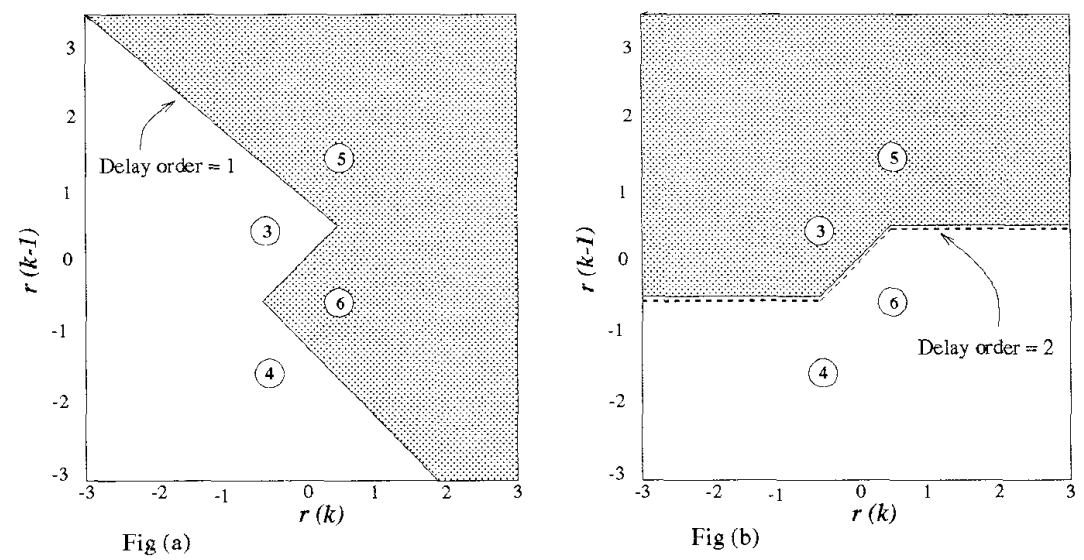

Figure 4: Realisation of decision boundary using subset RBF model.

Decision boundary for (Fig a) delay $d=1$, (Fig b) delay $d=2$.

By visual inspection of Figs. $4 \mathrm{~b}$ and $1 \mathrm{~b}$, it is clear that the subset model with centres $\left\{\mathbf{c}_{3}, \mathbf{c}_{4}, \mathbf{c}_{5}, \mathbf{c}_{6}\right\}$ is sufficient to realise the Bayesian boundary. Algorithm 1, however, picked all the centres to form the subset model. The reason for including centres $\left\{c_{1}, c_{2}\right\}$ and $\left\{c_{7}, c_{8}\right\}$ is that these two pairs of centres satisfy Eq. 15 in algorithm 1 and thus also define the asymptotic decision boundary. They are however unnecessary because the decision boundary formed using centres $\left\{\mathbf{c}_{3}, \mathbf{c}_{4}\right\}$ and $\left\{\mathbf{c}_{5}, \mathbf{c}_{6}\right\}$ are the same.

To minimise the inclusion of redundant centres, an additional condition is introduced in Eq. 15 to verify if the new centres under consideration affect decision boundary. If the decision boundary changes with the inclusion of the new centres, they will be accepted, otherwise ignored. By adding this condition, some redundant centres will not be included in the selected subset model. The algorithm for the improved version is as follows:

Algorithm 2 : Finding $U^{+}, U^{-}$

$$
\begin{gathered}
\mathbf{r}=\mathbf{c}_{i}+\left(\frac{\mathbf{c}_{j}-\mathbf{c}_{i}}{2}\right) \\
\text { if }\left[\begin{array}{l}
f(\mathbf{r})=0 \text { and } \\
\mathbf{c}_{i}=\min _{\mathbf{c}_{k} \in \mathrm{c}+\left\{\left\|\mathbf{r}_{0}-\mathbf{c}_{k}\right\|\right\} \text { and }} \\
f_{s}(\mathbf{r}) \neq 0 \\
\mathbf{c}_{i}-U^{+}, \mathbf{c}_{j} \rightarrow U^{-}
\end{array}\right] \\
f_{s}=\text { RBF model formed using } U^{+}, U^{-} \text {as centres. }
\end{gathered}
$$




\subsection{Subset model selection : some simulation results}

Simulations were conducted to select subset models from the full model used on channels $H 1(z)$ and $H 2(z)$. The feed forward order used was $m=4$, resulting in a full model with $N_{s}=2^{m+n_{a}-1}=64$ centres. Using SNR condition at $16 \mathrm{~dB}$, simulations were conducted to evaluate the performance of the subset RBF, full RBF and the linear Wiener equalisers for the two channels. The results are tabulated in Table $1 \mathrm{a}$ and $1 \mathrm{~b}$; The first column of each table indicates the delay order parameter, the second column shows the size of the subset model used while the third, fourth and fifth columns list the BER performance of the respective equalisers and the last column indicates if the decision boundary is linearly or not-linearly separable.

Our results indicate that the full RBF models' BER performance, for cases when the decision boundary is linearly separable, are normally better than those when the decision boundary is not linearly separable. This is not surprising since decision boundaries which are not linearly separable tend to be much more complicated and have more centres with different decision outputs near to each other. It was also observed that smaller-sized RBF subset models can be found for the case when the boundary is linearly separable, and their performance not significantly poorer than the full model's performance.

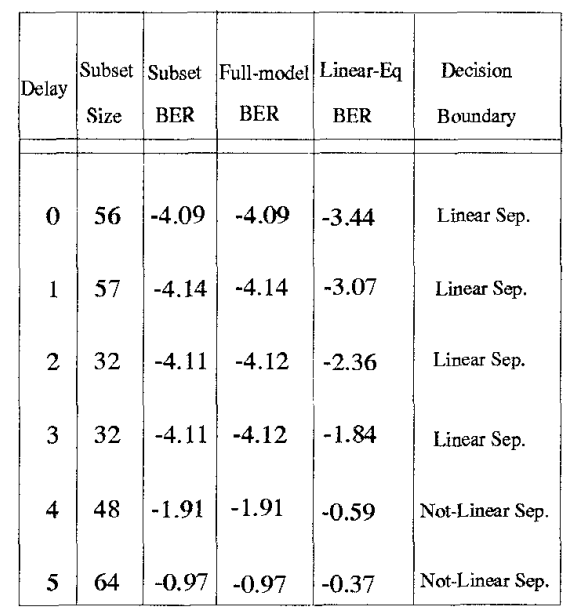

Table a : Channel H1(z)

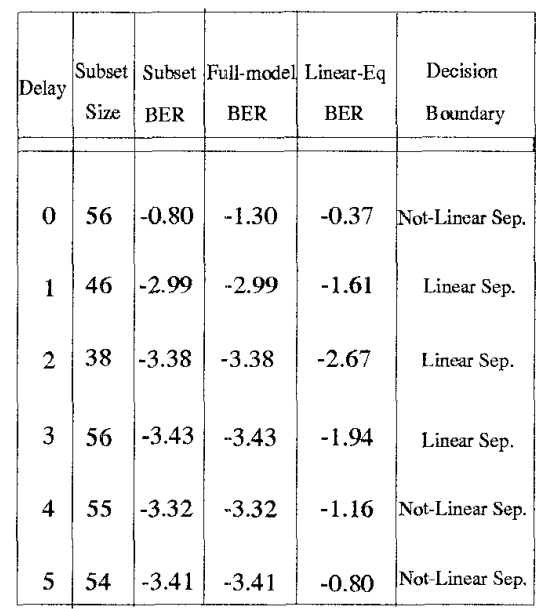

Table b : Channel H2(z)

Table 1: Comparing the performance of the full-size (64 centres) RBF equaliser, subset RBF equaliser and the Wiener equaliser for Channel $H 1(z)$ (Table $1 \mathrm{a}$ ) and Channel $H 2(z)$ (Table $1 \mathrm{~b}$ ) at $\mathrm{SNR}=16 \mathrm{db}$. 


\section{Conclusions}

This paper discusses the implementation of the RBF equaliser to realise the Bayesian solution. In particular, the effects of the delay order parameter on decision boundaries and BER performance is highlighted. We have showed that the attainable BER performance depends strongly on the delay order parameter and can be significantly different for various values of the delay order. To determine the optimum operating delay order parameter, a simple BER estimator for the RBF equaliser is proposed.

The implementation complexity of the RBF equaliser to realise the Bayesian solution is also discussed. To reduce some of the implementation complexity, we have introduced an algorithm to select subset model from the full RBF implementation. Our results indicate that that good subset models with no significant degradation in BER performance may be found.

\section{Acknowledgement}

We would like to thank Achilleas G Stogioglou of Edinburgh University for his many helpful comments and ideas during the development of this work.

\section{References}

[1] G.J.GIBSON, S.SIU, and C.F.N.COWAN, "The application of nonlinear structures to the reconstruction of binary signals", IEEE Trans. Signal Processing, vol. 39, no. 8, pp. 1877-1884, 1991.

[2] S.CHEN, B.MULGREW, and P.M.GRANT, "A clustering technique for digital communications channel equalization using radial basis function networks", IEEE Trans. Neural Networks, vol. 4, no. 4, pp. 570-579, 1993.

[3] C.P.CALLENDER and C.F.N.COWAN, "A comparison of six different non-linear equalisation techniques for digital communication systems", Proc. of the Seventh European Signal Processing Conference, pp. 1524$1527,1994$.

[4] S.U.H.QURESHI, "Adaptive equalization", Proc. IEEE, vol. 73, no. 9, pp. $1349-1387,1985$.

[5] B.HUGHES, "On the error probability of signals in additive white Gaussian noise", IEEE Trans. Information Theory, vol. 37, no. 1, pp. 151-155, 1991. 